\title{
Genuine Four Tangle for Four Qubit States
}

\author{
S. Shelly Sharma* and N. K. Sharma ${ }^{\dagger}$ \\ ${ }^{*}$ Depto. de Fisica, Universidade Estadual de Londrina, Londrina 86051-990, PR Brazil \\ ${ }^{\dagger}$ Depto. de Matematica, Universidade Estadual de Londrina, Londrina 86051-990, PR Brazil
}

\begin{abstract}
We report a four qubit polynomial invariant that quantifies genuine four-body correlations. The four qubit invariants are obtained from transformation properties of three qubit invariants under a local unitary on the fourth qubit.
\end{abstract}

Keywords: Four tangle, Polynomial invariants, multipartite entanlement

PACS: 3.67.Mn, 03.65.Ud

Two multipartite pure states are equivalent under stochastic local operations and classical communication (SLOCC) [1] if one can be obtained from the other with some probability using SLOCC. Attempts [2,3] to classify four-qubit pure states under SLOCC, have revealed that several entanglement classes contain a continuous range of strictly nonequivalent states, although with similar structure. In view of this, we proposed classification criteria [4] based on nature of multiqubit correlations in N-qubit pure states. In this article, we examine the three qubit invariants of four qubit states and derive higher degree invariants to quantify four and three-way correlations.

For a two qubit state, negative eigenvalue of partially transposed state operator is the invariant that distinguishes between a separable and an entangled state. In three qubit state space, two qubit subspace (for a selected pair of qubits) is characterized by a pair of two qubit invariants, while new two qubit invariants arise due to three body correlations in the composite space. The most important three qubit polynomial invariant is a degree four combination of two qubit invariants. The entanglement monotone constructed from this is Wootter's three tangle [5]. Four qubit states sit in the space $C^{2} \otimes C^{2} \otimes C^{2} \otimes C^{2}$ with three qubit subspaces for each set of three qubits. If there were no four body correlations, then three tangles should determine the entanglement of a four qubit state. When four body correlations are present, additional three qubit invariants that depend on four way negativity fonts [6] exist. Three qubit invariants, for a given set of three qubits, constitute a five dimensional space. In this article, we obtain four qubit invariants using transformation properties of three qubit invariants under a local unitary applied to the fourth qubit. One can continue the process to a higher number of qubits.

\section{FIVE THREE TANGLES}

Two qubit unitary invariants for pair of qubits $A_{1} A_{2}$ in the most general four qubit state

$$
\left|\Psi^{A_{1} A_{2} A_{3} A_{4}}\right\rangle=\sum_{i_{1} i_{2} i_{3} i_{4}} a_{i_{1} i_{2} i_{3} i_{4}}\left|i_{1} i_{2} i_{3} i_{4}\right\rangle ; \quad\left(i_{m}=0,1\right),
$$


are $D_{\left(A_{3}\right)_{i_{3}}\left(A_{4}\right)_{i_{4}}}^{00}, D_{\left(A_{3}\right)_{i_{3}}}^{00 i_{4}}-D_{\left(A_{3}\right)_{i_{3}}}^{01 i_{4}}, D_{\left(A_{4}\right)_{i_{4}}}^{00 i_{3}}-D_{\left(A_{4}\right)_{i_{4}}}^{01 i_{3}}, D^{0000}-D^{0100}, D^{0001}-D^{0101}$, where

$$
\begin{aligned}
D_{\left(A_{3}\right)_{i_{3}}\left(A_{4}\right)_{i_{4}}}^{00} & =\operatorname{det}\left[\begin{array}{ll}
a_{00 i_{3} i_{4}} & a_{01 i_{3} i_{4}} \\
a_{10 i_{3} i_{4}} & a_{11 i_{3} i_{4}}
\end{array}\right], D^{0 i_{2} 0 i_{4}}=\operatorname{det}\left[\begin{array}{ll}
a_{0 i_{2} 0 i_{4}} & a_{0, i_{2}+1,1, i_{4}+1} \\
a_{1 i_{2} 0 i_{4}} & a_{1, i_{2}+1,1, i_{4}+1}
\end{array}\right], \\
D_{\left(A_{3}\right)_{i_{3}}}^{0 i_{2} 0} & =\operatorname{det}\left[\begin{array}{ll}
a_{0 i_{2} i_{3} 0} & a_{0 i_{2}+1 i_{3} 1} \\
a_{1 i_{2} i_{3} 0} & a_{1 i_{2}+1 i_{3} 1}
\end{array}\right], D_{\left(A_{4}\right)_{i_{4}}}^{0 i_{2} 0}=\operatorname{det}\left[\begin{array}{ll}
a_{0 i_{2} 0 i_{4}} & a_{0 i_{2}+1,1 i_{4}} \\
a_{1 i_{2} 0 i_{4}} & a_{1 i_{2}+1,1 i_{4}}
\end{array}\right] .
\end{aligned}
$$

For qubits $A_{1} A_{2} A_{3}$ in $\left|\Psi^{A_{1} A_{2} A_{3} A_{4}}\right\rangle$, three qubit invariants

$$
\left(I_{3}^{A_{1} A_{2} A_{3}}\right)_{\left(A_{4}\right)_{i_{4}}}=\left(D_{\left(A_{4}\right)_{i_{4}}}^{000}-D_{\left(A_{4}\right)_{i_{4}}}^{010}\right)^{2}-4 D_{\left(A_{3}\right)_{0}\left(A_{4}\right)_{i_{4}}}^{00} D_{\left(A_{3}\right)_{1}\left(A_{4}\right)_{i_{4}}}^{00} ; \quad i_{4}=0,1 .
$$

quantify GHZ state like three-way correlations in three qubit state space. We examine the action of $\mathrm{U}^{A_{4}}=\frac{1}{\sqrt{1+|y|^{2}}}\left[\begin{array}{cc}1 & -y^{*} \\ y & 1\end{array}\right]$ on invariant $\left(I_{3}^{A_{1} A_{2} A_{3}}\right)_{\left(A_{4}\right)_{0}}$. The transformed invariant is a combination of five three qubit invariants that is

$$
\begin{aligned}
\left(I_{3}^{A_{1} A_{2} A_{3}}\right)_{\left(A_{4}\right)_{0}}^{\prime} & =\frac{1}{\left(1+|y|^{2}\right)^{2}}\left[\left(y^{*}\right)^{4}\left(I_{3}^{A_{1} A_{2} A_{3}}\right)_{\left(A_{4}\right)_{1}}-4\left(y^{*}\right)^{3} P_{\left(A_{4}\right)_{1}}^{A_{1} A_{2} A_{3}}\right. \\
& \left.+6\left(y^{*}\right)^{2} T_{A_{4}}^{A_{1} A_{2} A_{3}}-4 y^{*} P_{\left(A_{4}\right)_{0}}^{A_{1} A_{2} A_{3}}+\left(I_{3}^{A_{1} A_{2} A_{3}}\right)_{\left(A_{4}\right)_{0}}\right]
\end{aligned}
$$

Here prime denotes the transformed invariant and additional invariants are

$$
\begin{aligned}
& T_{A_{4}}^{A_{1} A_{2} A_{3}}= \frac{1}{6}\left(D^{0000}+D^{0001}+D^{0010}+D^{0011}\right)^{2} \\
&-\frac{2}{3}\left(D_{\left(A_{3}\right)_{0}}^{000}+D_{\left(A_{3}\right)_{0}}^{001}\right)\left(D_{\left(A_{3}\right)_{1}}^{000}+D_{\left(A_{3}\right)_{1}}^{001}\right) \\
&+\frac{1}{3}\left(D_{\left(A_{4}\right)_{0}}^{000}+D_{\left(A_{4}\right)_{0}}^{001}\right)\left(D_{\left(A_{4}\right)_{1}}^{000}+D_{\left(A_{4}\right)_{1}}^{001}\right) \\
&-\frac{2}{3}\left(D_{\left(A_{3}\right)_{0}\left(A_{4}\right)_{0}}^{00} D_{\left(A_{3}\right)_{1}\left(A_{4}\right)_{1}}^{00}+D_{\left(A_{3}\right)_{1}\left(A_{4}\right)_{0}}^{00} D_{\left.\left(A_{3}\right)_{0}\left(A_{4}\right)_{1}\right)}^{00}\right), \\
& P_{\left(A_{4}\right)_{i_{4}}}^{A_{1} A_{2} A_{3}}=\frac{1}{2}\left(D_{\left(A_{4}\right)_{i_{4}}}^{000}+D_{\left(A_{4}\right)_{i_{4}}}^{001}\right)\left(D^{0000}+D^{0001}+D^{0010}+D^{0011}\right) \\
&-\left(D_{\left(A_{3}\right)_{1}\left(A_{4}\right)_{i_{4}}}^{000}\left(D_{\left(A_{3}\right)_{0}}^{000}+D_{\left(A_{3}\right)_{0}}^{001}\right)+D_{\left(A_{3}\right)_{0}\left(A_{4}\right)_{i_{4}}}^{000}\left(D_{\left(A_{3}\right)_{1}}^{000}+D_{\left(A_{3}\right)_{1}}^{001}\right)\right) .
\end{aligned}
$$

Five three tangles, constructed from invariants $\left(I_{3}^{A_{1} A_{2} A_{3}}\right)_{\left(A_{4}\right)_{i_{4}}},\left(I_{3}^{A_{1} A_{2} A_{3}}\right)_{\left(A_{4}\right)_{i_{4}}}, P_{\left(A_{4}\right)_{0}}^{A_{1} A_{2} A_{3}}$, $P_{\left(A_{4}\right)_{1}}^{A_{1} A_{2} A_{3}}$, and $T_{A_{4}}^{A_{1} A_{2} A_{3}}$, capture the entanglement of $A_{1} A_{2} A_{3}$ due to three and four-way correlations. 


\section{GENUINE FOUR TANGLE}

Continuing the search for a four qubit invariant that detects genuine four-way correlations, we notice that when a selected $\mathrm{U}^{A_{4}}$ results in $\left(I_{3}^{A_{1} A_{2} A_{3}}\right)_{\left(A_{4}\right)_{0}}^{\prime}=0$, we have at hand a quartic equation. A quartic equation, $y^{4} a-4 b y^{3}+6 y^{2} c-4 d y+f=0$, in variable $y$ has associated polynomial invariants, $S=a f-4 b d+3 c^{2}$, cubic invariant $T=a c f-a d^{2}-b^{2} f+2 b c d-c^{3}$, and discriminant $\Delta=S^{3}-27 T^{2}$. Therefore, the degree eight polynomial invariant associated with $\left(I_{3}^{A_{1} A_{2} A_{3}}\right)_{\left(A_{4}\right)_{0}}^{\prime}=0$ is

$$
I_{(4,8)}^{A_{1} A_{2} A_{3} A_{4}}=3\left(T_{A_{4}}^{A_{1} A_{2} A_{3}}\right)^{2}+\left(I_{3}^{A_{1} A_{2} A_{3}}\right)_{\left(A_{4}\right)_{0}}\left(I_{3}^{A_{1} A_{2} A_{3}}\right)_{\left(A_{4}\right)_{1}}-4 P_{\left(A_{4}\right)_{0}}^{A_{1} A_{2} A_{3}} P_{\left(A_{4}\right)_{1}}^{A_{1} A_{2} A_{3}} .
$$

The discriminant is given by $\Delta=\left(I_{4}^{A_{1} A_{2} A_{3} A_{4}}\right)^{3}-27\left(J^{A_{1} A_{2} A_{3} A_{4}}\right)^{2}$, where

$$
J^{A_{1} A_{2} A_{3} A_{4}}=\operatorname{det}\left[\begin{array}{ccc}
\left(I_{3}^{A_{1} A_{2} A_{3}}\right) & P_{\left(A_{4}\right)_{1}}^{A_{1} A_{2} A_{3}} & T_{A_{4}}^{A_{1} A_{2} A_{3}} \\
P_{\left(A_{4}\right)_{1}}^{A_{1} A_{2} A_{3}} & T_{A_{4}}^{A_{1} A_{2} A_{3}} & P_{\left(A_{4}\right)_{0}}^{A_{1} A_{2} A_{3}} \\
T_{A_{4}}^{A_{1} A_{2} A_{3}} & P_{\left(A_{4}\right)_{0}}^{A_{1} A_{2} A_{3}} & \left(I_{3}^{A_{1} A_{2} A_{3}}\right)_{\left(A_{4}\right)_{0}}
\end{array}\right] .
$$

We may mention here that since there are four ways in which a given set of three qubits may be selected, $\Delta$ can be expressed in terms of different sets of three qubit invariants.

The four tangle $\tau_{(4,8)}=4\left|\left(12 I_{(4,8)}^{A_{1} A_{2} A_{3} A_{4}}\right)^{\frac{1}{2}}\right|$ quantifies 4-way correlations [7]. If four tangle is zero then transformation equations acquire a simpler form and yield four qubit invariants that quantify 3-way correlations. Invariant to quantify entanglement of a four qubit state having purely two qubit correlations can also be easily obtained. What is the utility of these polynomial invariants? Quantum entanglement distributed between distinct parties is a physical resource for practical quantum information processing. Polynomial invariants are used to construct entanglement monotones to quantify entanglement.

\section{ACKNOWLEDGMENTS}

Financial support from CNPq Brazil and FAEP UEL Brazil is acknowledged.

\section{REFERENCES}

1. F. Verstraete, J. Dehaene, B. DeMoor, and H. Verschelde, Phys. Rev. A 65, 052112 (2002).

2. L. Lamata, J. Leon, D. Salgado and E. Solano, Phys. Rev. A 75, 022318 (2007).

3. D. Li, X. Li, H. Huang, and X. Li, Quant. Inf. Comp. 9, 0778 (2009).

4. S. S. Sharma and N. K. Sharma, Phys. Rev. A 85, 042315 (2012).

5. V. Coffman, J. Kundu, and W. K. Wootters, Phys. Rev. A 61, 052306 (2000).

6. S. S. Sharma and N. K. Sharma, Phys. Rev. A 82, 052340 (2010).

7. S. S. Sharma and N. K. Sharma, Phys. Rev. A 87, 022335(2013). 